\title{
Benefícios dos procedimentos estéticos na melhora da autoestima
}

\author{
Benefits of aesthetic procedures improving self-esteem \\ Beneficios de los procedimientos estéticos rara mejorar la autoestima
}

Any Caroline de Morais Salomão

ORCID: https://orcid.org/0000-0002-3978-3562

Faculdade Independente do Nordeste, Brasil E-mail: anycms@hotmail.com

Lorena Lanusse de Oliveira Silva

ORCID: https://orcid.org/0000-0001-8900-6457

Faculdade Independente do Nordeste, Brasil E-mail: lanusse96@gmail.com Jeane Rocha Santos

ORCID: https://orcid.org/0000-0002-1398-3638

Faculdade Independente do Nordeste; Brasil E-mail: Jeane@ fainor.com.br

\begin{abstract}
Resumo
A autoestima é a maneira como o indivíduo se conhece e de como ele se relaciona com seu próprio corpo. A imagem física de um indivíduo é uma representação mental de acordo com sua perspectiva, não se restringindo somente as sensações e pensamentos, mas ao aspecto do seu próprio corpo. Este estudo teve como objetivo geral avaliar os benefícios dos procedimentos estéticos na melhora da autoestima. Trata-se de uma revisão de literatura. As buscas foram realizadas por meio de bases eletrônicas bibliotecas eletrônicas científicas online - Scielo, Bireme e Google instituições acadêmicas e LILACS (Literatura Científica e Tecnológica da América Latina e Caribe / BVS-Biblioteca Virtual em Saúde, através dos descritores autoestima, autoimagem e procedimentos estéticos. A análise dos resultados descreveu os impactos positivos na autoestima do indivíduo. Conclui-se que os procedimentos estéticos ajudam a elevar a autoestima, transformando um conceito positivo de si próprio e podendo enxergar sua beleza e qualidades. Ressalta-se a importância de mais estudos acerca do tema.
\end{abstract}

Palavra-chave: Autoestima; Autoimagem; Procedimentos estéticos.

\begin{abstract}
Self-esteem is the way an individual knows himself and how he relates to his own body. The physical image of an individual is a mental representation according to their perspective, not restricted to sensations and thoughts, but to the aspect of their own body. This study aimed to evaluate the benefits of aesthetic procedures in improving self-esteem. This is a literature review. Searches were performed through electronic databases online scientific electronic libraries - Scielo, Bireme and Google academic institutions and LILACS (Scientific and Technological Literature of Latin America and the Caribbean / VHL-Virtual Health Library, through the descriptors self-esteem, self-image and aesthetic procedures The analysis of the results described the positive impacts on the individual's self-esteem. It was concluded that aesthetic procedures help to raise self-esteem, transforming a positive self-concept and being able to see its beauty and qualities. about the topic.
\end{abstract}

Keywords: Self-esteem; Self-image; Aesthetic procedures.

\section{Resumen}

La autoestima es la forma en que un individuo se conoce a sí mismo y cómo se relaciona con su propio cuerpo. La imagen física de un individuo es una representación mental según su perspectiva, no restringida a sensaciones y pensamientos, sino al aspecto de su propio cuerpo. Este estudio tuvo como objetivo evaluar los beneficios de los procedimientos estéticos en la mejora de la autoestima. Ésta es una revisión de la literatura. Las búsquedas se realizaron a través de bases de datos electrónicas, bibliotecas electrónicas científicas en línea - Instituciones académicas Scielo, Bireme y Google y LILACS (Literatura Científica y Tecnológica de América Latina y el Caribe / BVS-Biblioteca Virtual en Salud, a través de los descriptores autoestima, autoimagen y estética procedimientos El análisis de los resultados describió los impactos positivos en la autoestima del individuo, se concluyó que los procedimientos estéticos ayudan a elevar la autoestima, transformando un autoconcepto positivo y pudiendo ver su belleza y cualidades sobre el tema.

Palabras clave: Autoestima; Auto imagen; Procedimientos estéticos. 


\section{Introdução}

De acordo com Gouveia et al. (2005), autoestima é considerada a maneira como o indivíduo se percebe, se avalia e se comporta, e é determinada pelos seus valores, crenças e culturas. Para Minayo (2009), o conceito de autoestima é definido como a maneira como o indivíduo se vê, como aceita a si mesmo, elege suas metas, seus sonhos e como valoriza o outro. Para Portinari (2000), a autoestima é a maneira como o indivíduo se conhece e de como ele se relaciona com seu próprio corpo. A imagem física de um indivíduo é uma representação mental de acordo com sua perspectiva, não se restringindo somente as sensações e pensamentos, mas ao aspecto do seu próprio corpo. Ainda segundo Caponi; Poli Neto (2002), o conceito de autoestima é: bem estar, felicidade e avaliação positiva de si. Uma autoestima elevada além de promover uma melhora no bem estar físico e mental, o indivíduo passa a ter relacionamentos melhores na sociedade e no ambiente de trabalho, além de se sentir mais satisfeito e consequentemente, mais determinado a alcançar seus sonhos e objetivos.

Para Neves (2012), a estética trata-se de uma ciência voltada para a beleza e para o despertar de algo belo dentro de cada indivíduo. Por isso, os espaços de estética foram criados para oferecer tratamentos no intuito de melhorar a aparência física e elevar a autoestima dos indivíduos. Fazer as pazes com o espelho, ter um cuidado com a pele, cabelos e corpo, por isso, a preocupação com a aparência é sinônimo de bem-estar. Os estudos mostram que os procedimentos estéticos produzem resultados positivos, o justifica sua importância na melhora da autoestima dos pacientes, nota-se então uma melhora da visão que eles têm de si mesmo e um aumento em seus relacionamentos sociais e interpessoais, além de uma melhora na qualidade de vida (Pereira et al., 2018).

Diante do exposto, justifica-se o presente estudo, pois buscar explicitar, através da literatura já existente, quais são os benefícios dos procedimentos estéticos na melhoria da autoestima dos indivíduos acometidos por disfunções estéticas.

O objetivo deste estudo é avaliar os benefícios dos procedimentos estéticos na melhora da autoestima.

\section{Metodologia}

Este estudo trata-se de uma revisão da literatura, que segundo Alves e Mazzotti (2002) propõe dois objetivos: estabelecer o contexto do problema e analisar as alternativas existentes na literatura para estabelecer o referencial teórico da pesquisa. Além disso, também possui as características de pesquisa descritiva exploratória, por se tratar de um assunto pouco explorado ou estudado (Gil, 2010). As buscas foram realizadas por meio de bases eletrônicas bibliotecas eletrônicas científicas online - Scielo, Bireme e Google instituições acadêmicas e LILACS (Literatura Científica e Tecnológica da América Latina e Caribe / BVS-Biblioteca Virtual em Saúde, através dos descritores autoestima, autoimagem e procedimentos estéticos. Os critérios de inclusão adotados foram: artigos relacionados aos temas e publicados entre 2010 e 2021 . Não foram aceitos artigos não relacionados ao tema e artigos publicados antes de 2005. Os resultados foram apresentados em um quadro contendo as seguintes informações: ano de publicação, autor, título, objetivo, métodos, resultados. Em seguida fez-se uma análise descritivas à luz dos artigos estudados, comparando os resultados obtidos com os resultados de outras pesquisas desenvolvidas sobre o tema. O estudo respeitou as leis dos direitos autorais que, de acordo a lei número 9.610, de fevereiro de 1998, assegura que as literaturas propostas no estudo foram referenciadas corretamente, sem cópias ou plágios.

\section{Resultados e Discussão}

Inicialmente foram selecionados 20 artigos, porém destes apenas 10 tinham relação com o tema. Destes 4 foram descartados por não se adequarem completamente aos critérios de inclusão e exclusão supracitados. Ao final da filtragem restaram 6 artigos. Realizou-se a criação de um quadro que possibilitou organizar os dados dos artigos em: título, autor e ano, objetivo, métodos e resultados, conforme o Quadro 1 abaixo: 
Quadro 1 - Apresentação dos artigos segundo título, autor (es), ano de publicação, objetivos, métodos e principais resultados. Vitória da Conquista, Bahia, Brasil. 2021.

\begin{tabular}{|c|c|c|c|c|}
\hline Título & Autor $\backslash$ Ano & Objetivos & Métodos & Resultados \\
\hline $\begin{array}{l}\text { Auto-estima e auto- } \\
\text { imagem: a relação com } \\
\text { a estética }\end{array}$ & $\begin{array}{l}\text { Monique Flavia; } \\
\text { Silva Margara; } \\
\text { Baggio, Laércio } \\
\text { (2010) }\end{array}$ & $\begin{array}{l}\text { Esta pesquisa teve como objetivo } \\
\text { esclarecer conceitos de auto- } \\
\text { estima, auto-imagem e a relação } \\
\text { destes com a } \\
\text { Estética, }\end{array}$ & $\begin{array}{c}\text { Pesquisa } \\
\text { bibliográfica do } \\
\text { tipo explicativa }\end{array}$ & $\begin{array}{l}\text { Caracterizando-se como uma pesquisa } \\
\text { bibliográfica do tipo explicativa, por ter } \\
\text { sido elaborada a partir de consultas em } \\
\text { livros, artigos científicos e sites nas bases } \\
\text { de dados. }\end{array}$ \\
\hline $\begin{array}{c}\text { Avaliação da } \\
\text { autoestima das } \\
\text { mulheres que realizam } \\
\text { procedimentos estéticos } \\
\text { nas clínicas escolas da } \\
\text { Unisul Pedra Branca }\end{array}$ & $\begin{array}{l}\text { Meyer, daniela; } \\
\text { goulart, gabbiela; } \\
\text { (2018) }\end{array}$ & $\begin{array}{l}\text { O objetivo do estudo foi avaliar a } \\
\text { autoestima das mulheres que } \\
\text { realizam procedimentos estéticos } \\
\text { nas clínicas escolas da Unisul- } \\
\text { Pedra branca. }\end{array}$ & $\begin{array}{l}\text { Estudo analítico, } \\
\text { observacional, } \\
\text { transversal com } \\
\text { abordagem } \\
\text { quantitativa. }\end{array}$ & $\begin{array}{l}\text { A coleta de dados do presente estudo foi } \\
\text { realizada no mês de junho de } 2019 \text {, } \\
\text { sendo aplicados } 86 \text { questionários, que } \\
\text { foram preenchidos por mulheres que } \\
\text { realizaram procedimentos estéticos nas } \\
\text { clínicas escolas da Unisul-Pedra Branca. } \\
\text { Destes questionários } 03 \text { foram excluídos } \\
\text { por estarem incompletos ou com mais de } \\
\text { uma alternativa assinalada, totalizando a } \\
\text { inclusão de } 83 \text { indivíduos no estudo }\end{array}$ \\
\hline $\begin{array}{l}\text { A relevância dos } \\
\text { cuidados corporais para } \\
\text { a autoestima das } \\
\text { pessoas: uma revisão } \\
\text { da literatura }\end{array}$ & $\begin{array}{l}\text { Eduardo, maria } \\
\text { aparecida; } \\
\text { Ferreira, keila } \\
\quad(2018)\end{array}$ & $\begin{array}{l}\text { Pensando nisso, este trabalho tem } \\
\text { o objetivo de investigar na } \\
\text { literatura, estudos sobre a } \\
\text { influência da aparência na vida das } \\
\text { pessoas, apresentando } \\
\text { informações a fim de levar a } \\
\text { compreensão sobre o que motiva } \\
\text { as pessoas a procurarem os } \\
\text { serviços de estética. }\end{array}$ & $\begin{array}{l}\text { uma revisão da } \\
\text { literatura }\end{array}$ & $\begin{array}{l}\text { Mediante aos textos estudados, foi } \\
\text { constatado que a beleza está associada à } \\
\text { saúde do corpo em harmonia com a } \\
\text { mente, pois o equilíbrio entre este } \\
\text { proporciona satisfação pessoal que } \\
\text { reflete em vários níveis de socialização } \\
\text { do indivíduo. }\end{array}$ \\
\hline $\begin{array}{l}\text { Uma reflexão sobre a } \\
\text { influência da estética } \\
\text { na auto estima, } \\
\text { Auto-motivação e bem } \\
\text { estar do ser humano. }\end{array}$ & $\begin{array}{l}\text { Borba, tamila; } \\
\text { thives, fabiana ; } \\
\text { (2011) }\end{array}$ & $\begin{array}{l}\text { Já é sabido que a vaidade é uma } \\
\text { grande característica do povo } \\
\text { brasileiro, e o campo da área } \\
\text { estética é bastantea mplo, por este } \\
\text { motivo este trabalho teve como } \\
\text { objetivo questionar quais são os } \\
\text { procedimentos e tratamentos de } \\
\text { embelezamento que mais } \\
\text { influenciam na automotivação e } \\
\text { auto estima dos seres humanos. }\end{array}$ & $\begin{array}{l}\text { Pesquisa } \\
\text { bibliográfica, } \\
\text { qualitativa e } \\
\text { exploratória. }\end{array}$ & $\begin{array}{l}\text { Além disso, a pesquisa evidenciou que } \\
\text { homens e mulheres prefeririam } \\
\text { procedimentos estéticos a cirurgias } \\
\text { plásticas, caso ambas viessem a } \\
\text { apresentar os mesmos resultados. Tal } \\
\text { visão contrasta com dados que apontam } \\
\text { o brasil como sendo um país de } \\
\text { crescimento constante, no que tange à } \\
\text { realização de cirurgias plásticas, e eleva } \\
\text { a importância dos tratamentos estéticos } \\
\text { na busca pela autoestima e imagem } \\
\text { corporal ideal. }\end{array}$ \\
\hline $\begin{array}{l}\text { A Importância dos } \\
\text { Procedimentos } \\
\text { Estéticos na } \\
\text { Autoestima da Mulher }\end{array}$ & $\begin{array}{l}\text { Da Silva, } \\
\text { Roseneide; } \\
\text { Barro, Zamia. } \\
\quad(2020)\end{array}$ & $\begin{array}{l}\text { Analisar o } \quad \text { impacto de } \\
\text { procedimentos estéticos, em } \\
\text { especial, do design de sobrancelha } \\
\text { na autoestima da mulher }\end{array}$ & $\begin{array}{l}\text { Revisão de } \\
\text { literatura }\end{array}$ & $\begin{array}{l}\text { Os achados dessa pesquisa demonstram } \\
\text { que processos dermatológicos } \\
\text { cosméticos, como preenchedores de } \\
\text { rugas injetáveis, toxina botulínica para } \\
\text { linhas finas, recapeamento da pele com } \\
\text { laser e outras fontes de luz, peelings } \\
\text { químicos, mesoterapia e outros } \\
\text { procedimentos estéticos, aumentam a } \\
\text { confiança e melhora a vida social }\end{array}$ \\
\hline $\begin{array}{c}\text { Relação dos } \\
\text { procedimentos estéticos } \\
\text { com satisfação da } \\
\text { autoimagem corporal e } \\
\text { autoestima de mulheres }\end{array}$ & $\begin{array}{l}\text { Pinheiro, talita; } \\
\text { Piovezan, } \\
\text { nayane; Batista, } \\
\text { Helder; Muner, } \\
\text { Luana, (2020) }\end{array}$ & $\begin{array}{l}\text { Essa pesquisa teve por objetivo } \\
\text { verificar a relação dos } \\
\text { procedimentos estéticos com o } \\
\text { grau de satisfação da imagem } \\
\text { corporal e autoestima de mulheres. }\end{array}$ & Estudo amostral & $\begin{array}{l}\text { Foi identificada uma associação positiva } \\
\text { e significativa entre a autoestima e } \\
\text { satisfação com a imagem corporal, de } \\
\text { forma que quanto mais satisfeitas as } \\
\text { mulheres estão com o seu peso, maior } \\
\text { tende a ser seus níveis de autoestima. }\end{array}$ \\
\hline
\end{tabular}

Fonte: Dados da pesquisa.

A autoestima é considerada a forma como os indivíduos percebem, avaliam e se comportam e é determinada por seus valores, crenças e cultura. (Gouveia et al., 2005). Para Caponi e Poli Neto (2002), os conceitos de autoestima são: felicidade, bem-estar e autoavaliação positiva. Elevada autoestima, além de promover a melhoria da saúde física e mental, os indivíduos passam a construir melhores relações na sociedade e no ambiente de trabalho, além de se sentirem mais satisfeitos e portanto, mais determinados a alcançar seus sonhos e objetivos. De acordo com Duclos et al. (2006), a autoestima é considerada o 
sentimento que o indivíduo tem em relação a sua avaliação entre seu autoconhecimento e o seu ego ideal. Para Hidalgo e Palacios (2004), a autoestima é a autoavaliação que o indivíduo faz, do quanto ele se valoriza e se considera competente. Trata-se da forma como uma pessoa sente-se em relação ao que ela é.

Os centros de estética foram criados para oferecer tratamentos que busquem melhorar a aparência e consequentemente, a autoestima. Os cuidados com a pele, cabelos e corpo, segundo a autora, é sinônimo de saúde e felicidade. Após a revisão de literatura, percebeu-se que os procedimentos estéticos tem o poder de mudar a autoestima das pessoas, tanto homens ou mulheres, aumentando e causando uma sensação de bem estar (Neves, 2012)

A autoestima tem sido pautada como uma importante referência de saúde mental, tendo relação com a autoimagem (Floriani et al., 2010). Estando relacionada a autoconfiança, pois a pessoa com boa autoestima torna-se mais confiante diante das decisões a serem tomadas (Andrade, et al. 2009). Se desenvolve durante a vida, gerada a partir dos elogios, carinho, atenção e até mesmo de repreensão e críticas no âmbito familiar, escolar, social entre outros (Guilhardi, 2002).

Um estudo realizado por Ferreira et al. (2016) mostrou o quanto há relação entre tratamento estético e autoestima, sendo capaz de alterar a qualidade de vida feminina e masculina. Utilizando uma escala de autoestima, Santos et al (2019) demostrou que aspectos psicossociais do período pré e pós-operatório indicam que a aceitação aos procedimentos cosméticos pósoperatórios melhorou a autoestima. Há uma grande acessibilidade às informações com variedade de novidades estéticas com valores acessíveis a todas as classes sociais. Conquistando um público mais exigente e informado que deseja expandir suas práticas de maturidade (Vigarello, 2006).

Para algumas pessoas, a cirurgia estética, é uma forma de superar o poder avassalador da malformação, da imagem social e do aumento da autoestima. Entende-se, portanto, que o mercado brasileiro de cirurgia plástica tem grande importância na vida de cada ser humano e que os profissionais buscam ser cada vez mais qualificados de modo a atender essa demanda (Saraiva, 2019).

Desta forma, ao realizar comparações com os padrões de beleza, advém as distorções na percepção da imagem corporal, gerando um grave fracasso que levando a perda da autoestima e a inseguranças com a própria aparência (Secchi et al., 2009). Para alcançar esses objetivos de beleza, principalmente entre as mulheres, cresce cada vez mais a busca por programas de atividade física e por atendimentos estéticos (Vieira et al., 2016). Para Borba et al. (2011), na atualidade o bem estar físico e mental são fatores decisivos para obter melhora da autoestima, e esse bem-estar pode ser encontrado nos tratamentos estéticos.

Ressalta-se a importância da valorização dos profissionais de estética, além dos serviços prestados por essa categoria. Desejando mostrar que a estética não apenas muda o exterior das pessoas com as quais trabalha, mas também a forma como cada qual se sente e se vê após realizar tratamentos estéticos (Pereira et al., 2018). Profissionais da Estética que buscam o melhor do seu serviço e para seus clientes, também buscam aperfeiçoar no melhor bem estar e comodidade para tal. Por meio da estética, o profissional também busca entregar felicidade, saúde e qualidade de vida. A Estética Humanizada traz valorização ao paciente, procurando reforçar os cuidados e atenção (Aguiar, 2020).

Entretanto, é importante lembrar que os procedimentos estéticos colaboram e auxiliam, mas, não devem ser a única fonte de busca pela felicidade. Deve-se considerar outros valores, como o amor próprio, o equilíbrio emocional, sonhos e realizações que não são necessariamente sempre ligados à questão estética (Nogueira, 2021). Outro aspecto que relacionam a imagem corporal ao interesse pela cirurgia plástica é a orientada pela aparência, ou o investimento nela, que significa o que os indivíduos valorizam a sua aparência e o que eles prestam atenção nesse aspecto.

Algumas pesquisas também apontam ainda a insatisfação específica pode influenciar a decisão de se submeter à cirurgia estética, o que não ocorre quando há insatisfação corporal global (Sarwer et al., 1998).

Quando pensamos na relação entre qualidade de vida e beleza é preciso entender que cada indivíduo deseja por objetivos diferentes, e tem expectativas diferentes no alcance da beleza. Um exemplo extremo são as modelos de passarela ou manequim 
que tem exigências rígidas quanto às medidas corporais. Nesse caso é preciso maior cuidado, pois a qualidade de vida pode ser prejudicada devida tamanha cobrança (Lisboa 2016). Para Cash (1990), a imagem corporal e faciais pode ser definida como ideias, pensamentos e sentimentos sobre seu corpo, seu rosto e experiências corporais. Devido ao sucesso das redes sociais elas são o principal método do desenvolvimento da busca pela beleza e perfeição, da divulgação de novos procedimentos e cirurgias estéticas, novas dietas mirabolantes e remédios que prometem fazer milagres pelo seu corpo (Araújo, 2020).

O mercado oferece produtos e procedimentos estéticos com resultados satisfatórios e imediatos. Para o sucesso no tratamento de qualquer disfunção estética seja ela gordura localizada, flacidez, FEG, estrias, depende essencialmente do conhecimento que o profissional tem da anatomia, fisiologia e da patologia a ser tratada, bem como sua qualificação e atualização, pois assim poderá escolher os tratamentos adequados, obtendo excelentes resultados (Schmitz, 2010).

A estética também pode ajudar na redução de medidas e edemas, estimular a circulação sanguínea, o metabolismo e as respostas neuromusculares, harmonizar os contornos corporais e diminuindo a ansiedade e a depressão, contribuindo para o aumento da autoestima e da qualidade de vida. (Santos, 2010). Um exemplo de expressão é a moda que não deve ser vista apenas como roupas como muitos pensar, ela engloba a cultura, língua, modo de agir, decoração e vários outros fatos importantes (Freitas et al., 2010). Ao se notar pelas faixas etárias, tanto jovens como idosos, é possível ver que existe uma compreensão no que diz respeito ao ser belo e as diferentes formações sociais (Loureiro, 2003).

Tendo em vista a importância dos procedimentos estéticos para a estabilidade emocional e sociopsicológica dos pacientes submetidos a esses procedimentos, estudos de desenvolvimento têm comprovado que sua eficácia para a autoestima pessoal é muito importante (Schultheisz, 2015). Os resultados do estudo sobre as pontuações apoiam o argumento embelezamento estético da cirurgia refrativa e sua com saúde, uma vez que a satisfação com a aparência era aparente após a cirurgia, caracterizando a narrativa da vida relacionada à estética (Ferreira, 2010).

Saúde, estética e redes de localização foram identificadas como as motivações mais fortes para ir à academia, com uma quarta motivação importante para praticar atividade física: culpa, resultante da responsabilidade do indivíduo pelo aparecimento de seus defeitos corporais e imperfeições sendo entendidas como produto de negligência e negligência (Castro, 2001).

Certas técnicas praticadas pela esteticista, como uma simples massagem ou uma drenagem linfática, permitem evitar que tragam bem-estar físico e mental, mantendo o equilíbrio e reduzindo as despesas de medicamentos, fichas médicas e que o indivíduo tenha uma vida saudável e estável para suas atividades diárias (Orasmo, 2015).

No Brasil, a busca por um padrão de beleza é corrida contra o tempo, a cada minuto há novas informações, um novo produto, um novo tratamento estético, uma nova tendência, um novo estilo. Já sabemos que vaidade é grande para o povo brasileiro, e o campo da estética é amplo. Salões de beleza, clínicas estéticas, atividades, como o alívio do estresse da vida moderna, moradores da cidade, academias e modo de condicionamento físico, mova aquele que respira vigor e está aberto a notícias e inovação (Filgueras, 2018).

\section{Conclusão}

Diante do exposto conclui-se que os procedimentos estéticos ajudam a elevar a autoestima, transformando um conceito positivo de si próprio e podendo enxergar sua beleza e qualidades. A busca de procedimentos estéticos de alta tecnologia e personalizados proporcionou bem estar tanto entre as mulheres tanto quanto aos homens. É evidente que a vaidade que tais qualidades sejam reconhecidas e admiradas originam mudanças e resultados que contribuíram muito para que as pessoas passem a se sentir melhor consigo mesmas. "cuidar da beleza é cuidar da saúde, influenciando na autoestima e na qualidade de vida".

Ademais, ressalta-se a importância de novos estudos que possam analisar como cada procedimento estético e seus resultados ajudam na melhora da autoestima e, consequentemente, na qualidade de vida dos indivíduos. 


\section{Referências}

Aguiar, V. (2020). Euro Anglo | A importância da estética e sua humanização para o bem estar. Euro Anglo | Educar e Profissionalizar. https://euroanglocursos.com.br/blog/a-importancia-da-estetica-e-sua-humanizacao-para-o-bem-estar

Amanda, P., \& Bitencourt, B. (2018). Auto estima e bem estar pós tratamentos de rejuvenescimento facial. canva.com/pt_br/q/registro/brochuras/. http://canva.com/pt_br/q/registro/brochuras/

Araújo, r., silva, m., melo, c., \& câmara, c. (2020). Instagram e saúde mental: a influência dos padrões de beleza na autoestima de jovens. Encontro de extensão, docência e iniciação científica (EEDIC), 7. Recuperado de http://publicacoesacademicas.unicatolicaquixada.edu.br/index.php/eedic/article/view/4152

Arroyo, C. A imagem corporal e a autoestima de mulheres que procuram atendimento estético e exercícios físicos em spa - pdf Download grátis. Apresentamoslhe umas ferramentas confortáveis e gratuitas para publicar e compartilhar informações. https://docplayer.com.br/5723361-A-imagem-corporal-e-a-autoestimade-mulheres-que-procuram-atendimento-estetico-e-exercicios-fisicos-em-spa.html

Auricchio, A. M. Procedimentos estéticos: percepção do cliente quanto ao esclarecimento para a tomada de decisão. Biblioteca Digital de Teses e Dissertações da USP. https://www.teses.usp.br/teses/disponiveis/7/7131/tde-21122006-105242/publico/Ana_Aurichio.pdf

$\begin{array}{llllll}\text { Auto-imagem } & \text { S } & \text { Sentimento } & \text { de } & \text { Constrangimento. } & \text { Psico, }\end{array}$ https://revistaseletronicas.pucrs.br/ojs/index.php/revistapsico/article/view/1393 de

Barbosa, M. A Estética Na Busca Pela Qualidade De Vida. Hospedando 24Horas - Hospedagem de Qualidade. http://bibliotecaatualiza.com.br/arquivotcc/mp69/ALVES-elisangela-jesus-da-gama-BARBOSA-monique-de-jesus-braga.pdf

Braggio, L. Auto-Estima E Auto-Imagem: A Relação Com A Estética - PDF Free Download. Apresentamos-lhe umas ferramentas confortáveis e gratuitas para publicar e compartilhar informações. https://docplayer.com.br/5882778-Auto-estima-e-auto-imagem-a-relacao-com-a-estetica.html

Caponi, S. (2007). A medicalização da beleza. SciELO - Brasil. https://www.scielo.br/j/icse/a/bRhg3sPzPVTZZ4Wpvp53wmj/abstract/?lang=pt

Castro, A. L. (2001). Culto ao corpo e sociedade: midia, cultura de consumo e estilos de vida. CES - Centre for Social Studies. https://ces.uc.pt/lab2004/inscricao/pdfs/painel24/analuciacastro.pdf

Desenvolvimento Psicológico e Educação: Volume 1: Psicologia Evolutiva (2a ed.). (2004). Penso.

Ferreira, K. A RELEVÂNCIA DOS CUIDADOS CORPORAIS PARA A AUTOESTIMA DAS PESSOAS: uma revisão da literatura - PDF Download grátis. Apresentamos-lhe umas ferramentas confortáveis e gratuitas para publicar e compartilhar informações. http://docplayer.com.br/211337785-A-relevancia-doscuidados-corporais-para-a-autoestima-das-pessoas-uma-revisao-da-literatura.html

Ferreira, Z. A. B., \& Matins, R. d. S. G. (2020). A Importância dos Procedimentos Estéticos na Autoestima da Mulher. Id online, 14(i53.2807), 12. https://idonline.emnuvens.com.br/id/article/view/2807

Filgueiras, N. (2018). O crescimento e valorização do mercado de estética no brasil | filgueiras | repositório de trabalhos de conclusão de curso. Portal UNIFACIG de Publicações. http://pensaracademico.facig.edu.br/index.php/repositoriotcc/article/view/1009/895

Floriane, F. auto estiam $e$ auto imagem: a relação com $a$ estetica. IIS http://siaibib01.univali.br/pdf/Flavia\%20Monique\%20Floriani,\%20Márgara\%20Dayana\%20da\%20Silva\%20Marcante.pdf

Freitas, C. M. (2010). O padrão de beleza corporal sobre o corpo feminino mediante o IMC. SciELO, 23(3), 16. https://doi.org/10.1590/S180755092010000300010

Guilhardi, H. J. (2002). Instituto de Terapia por Contingências de Reforçamento. ITCR-Campinas - Instituto de Terapia por Contingências de Reforçamento Instituto de Terapia por Contingências de Reforçamento. https://itcrcampinas.com.br/pdf/helio/Autoestima_conf_respons.pdf

Gois, T. (n.d.). Influência Da Estética Na Autoestima E Bem Estar Do Ser Humano - PDF Download grátis. Apresentamos-lhe umas ferramentas confortáveis e gratuitas para publicar e compartilhar informações. https://docplayer.com.br/49184985-Influencia-da-estetica-na-autoestima-e-bem-estar-do-ser-humano.html

Goulart, G. (n.d.). Aliação Da Autoestima Das Mulheres Que Realizam Procedimentos Estéticos Nas Clínicas Escolas Da Unisulpedra Branca. Runa Repositório Universitário Aa Anima: inicial. https://repositorio.animaeducacao.com.br/bitstream/ANIMA/7939/1/Avaliação\%20da\%20autoestima\%20das\%20mulheres\%20que\%20realizam\%20procedim entos\%20estéticos\%20nas\%20Clínicas\%20Escolas\%20da\%20Unisul-\%20Pedra\%20Branca.pdf

Laourentino, L. Estetica Facial e Corporal: uma revisão bibliografica. IIS Windows http://siaibib01.univali.br/pdf/Delourdes\%20Schafascheck\%20Schmitz,\%20Lucia\%20Laurentino.pdf

Loureiro, I. (2003). Sobre as várias noções de estética em Freud| Pulsional rev. psicanál;16(175): 23-32, nov. 2003. | INDEXPSI. Pesquisa | Portal Regional da BVS. https://pesquisa.bvsalud.org/portal/resource/pt/psi-27794

Medeiros, F., Bitencourt, B., \& Pereira, A. (2018). Autoestima e bem estar pós-tratamentos de rejuvenescimento facial. https://repositorio.animaeducacao.com.br/handle/ANIMA/7906. https://repositorio.animaeducacao.com.br/handle/ANIMA/7906

Minayo, M. C. (2009). Intervenção visando a auto-estima e qualidade de vida dos policiais civis do Rio de Janeiro. SciELO - Brasil. https://www.scielo.br/j/csc/a/FmHDf4xy7cTKKk4Cch3qMCg/abstract/?lang=pt

Miyake, g., miyake, g., \& miyake, e. (2021). Cirurgia refrativa, muito além da satisfação e da aparência: uma revisão integrativa. Revista Científica Multidisciplinar Núcleo Do Conhecimento:, 7(6), 7. https://www.nucleodoconhecimento.com.br/saude/cirurgia-refrativa 
Research, Society and Development, v. 10, n. 16, e590101624308, 2021

(CC BY 4.0) | ISSN 2525-3409 | DOI: http://dx.doi.org/10.33448/rsd-v10i16.24308

Neves, M. (2015). Nutrição Estética e Nutricosméticos: Uma Abordagem Prática. AS Sistemas.

Nogueira, O. (2021). Bella Center Cosméticos - A influência da estética na autoestima e bem-estar do ser humano. Bella Center Cosméticos. https://www.bellacentercosmeticos.com.br/news-post.php?post=5

Oliveira, R. (2017). Tratamento estético e o conceito do belo | caderno de graduação - ciências biológicas e da saúde - unit - pernambuco. Periódicos Grupo Tiradentes. https://periodicos.set.edu.br/facipesaude/article/view/4064

Orasmo, B. (2021). trabalho de estetica PIM VII. Passei Direto. https://www.passeidireto.com/arquivo/101006863/trabalho-de-estetica-pim-vii/6

Padilha, C. R. A relação entre o conceito de beleza na construção da autoestima e o desenvolvimento do transtorno dismórfico corporal - Brasil Escola. Monografias Brasil Escola. https://monografias.brasilescola.uol.com.br/psicologia/a-relacao-entre-o-conceito-de-beleza-na-construcao-da-auto-estima-e-odesenvolvimento-do-transtorno-dismorfico-corporal.htm

Pinheiro, T., Piovezan, N., Batista, H., \& Muner, L. (2020). Relação Dos Procedimentos Estéticos Com Satisfação Da Autoimagem Corporal E Autoestima De Mulheres. Revista Cathedral, 2(1). Recuperado de http://cathedral.ojs.galoa.com.br/index.php/cathedral/article/view/106

Rezer, J. (2016, 19 de setembro). procedimentos estéticos não invasivos para o tratamento da gordura localizada e a lipoaspiração | salão do conhecimento. portal de eventos unijuí. https://www.publicacoeseventos.unijui.edu.br/index.php/salaoconhecimento/article/view/6680

Santos, C. G., Almeida, A. C., Carvalho, A., Costa, E., \& Junior, A. (2018). A atuação da estética no sistema único de saúde (sus). Revista Iniciação Cientifica, 8(2), 13. http://periodicos.unincor.br/index.php/iniciacaocientifica/article/view/5478

Saraiva, P. (2019). Influência Da Estética Na Auto Estima. Drpaulo. https://www.clinicapaulosaraiva.com.br/post/influência-da-estética-na-auto-estima

Sarwer, D. (2002). Awareness and identification of body dysmorphic disorder by aesthetic surgeons: results of a survey of american society for aesthetic plastic surgery members. https://pubmed.ncbi.nlm.nih.gov/19332010/

Schultheisz, T. (2015). Autoestima, conceitos correlatos e avaliação | Semantic Scholar. Semantic Scholar | AI-Powered Research Tool https://www.semanticscholar.org/paper/Autoestima,-conceitos-correlatos-e-avaliação-Schultheisz-Aprile/ec5e1614fe0626b23162222a018339967d6fd3b7

Soaigher, K. A., Acencio, F. R., \& Cortez, D. A. G. (2016). O poder da vaidade e do autocuidado na qualidade de vida. Cinergis, 18(1). https://doi.org/10.17058/cinergis.v18i1.8218

Souza, M., Souza, M., \& Paterno, J. (2021). Avaliação da aplicação de ácido hialurônico e radiofrequência na redução de rugas peribucais e melhoria da autoestima. Revista Brasileira De Estética Científica, 2(1), 102. https://doi.org/10.47483/abesci.v2i1

Thives, F. (n.d.). Uma Reflexão Sobre A Influência Da Estética Na Auto Estima | PDF | Estética | Beleza. Scribd. https://pt.scribd.com/document/343654520/Uma-Reflexao-Sobre-a-Influencia-Da-Estetica-Na-Auto-Estima

Vigarello, G. (2006). História da Beleza. Ediouro.

Yamasaki, V. (2013). O Consumo de Cirurgia Estética: a Influência da Autoestima e do Materialismo. Revista de Gestão em Sistemas de Saúde, 2(2), 23. https://doi.org/10.5585/rgss.v2i2.77 